\section{Highlights and the perception of glossiness}

\author{
JACOB BECK and SLAVA PRAZDNY \\ Computer Vision Laboratory \\ University of Maryland, College Park, Maryland 20742
}

Beck (1972) published a picture of a vase with and without highlights. The vase with highlights looked glossy. The vase without highlights looked matte. There are two alternative hypotheses concerning the perception of glossiness. One hypothesis is that the perception of glossiness, like the darkening due to contrast, is a spatially local perceptual quality that results from the presence of highlights. The second hypothesis is that the perception of glossiness is the result of a perceptual inference that a surface reflects light specularly based upon the information provided by highlights. The perception of glossiness is the result of both sensations and an inference based on past experience that causes the entire surface to appear glossy. Helmholtz explained the effects of surface texture and finish on the perception of color in these terms (Graham \& Brown, 1965). The two hypotheses are examined by studying how the size, brightness, orientation, the placement of highlights, and the intensity gradient on the vase surface affect the perception of glossiness.

The rightmost photograph in A of Figure 1 reproduces a digitized picture of the glossy vase in which all highlights were removed. In the left photographs, single highlights are superimposed on the digitized vase pictures. The positions of these highlights correspond to the positions of actual highlights originally on the vase. The vertical and horizontal intensity profiles of these highlights have the shapes of an inverted $\mathrm{V}$ and were chosen for computational simplicity. Forbus (Note 1) has shown that the intensity profile of a highlight is not critical as long as it is not discontinuous. The highlights make their surrounding areas look shiny. They fail, however, to make the entire vase look shiny. In B, two highlights are shown. The effects of the individual highlights combine and make the upper part of the vase look shiny. The lower part of the vase, however, continues to look dull. Highlights have a local effect that makes surrounding areas of the vase appear glossy. They do

The support of the National Science Foundation under Grant MCS-79-23422 is gratefully acknowledged, as is the help of Janet Salzman in preparing this paper. J. Beck was on sabbatical leave from the University of Oregon. Reprints may be obtained from J. Beck, Department of Psychology, University of Oregon, Eugene, Oregon 97403.
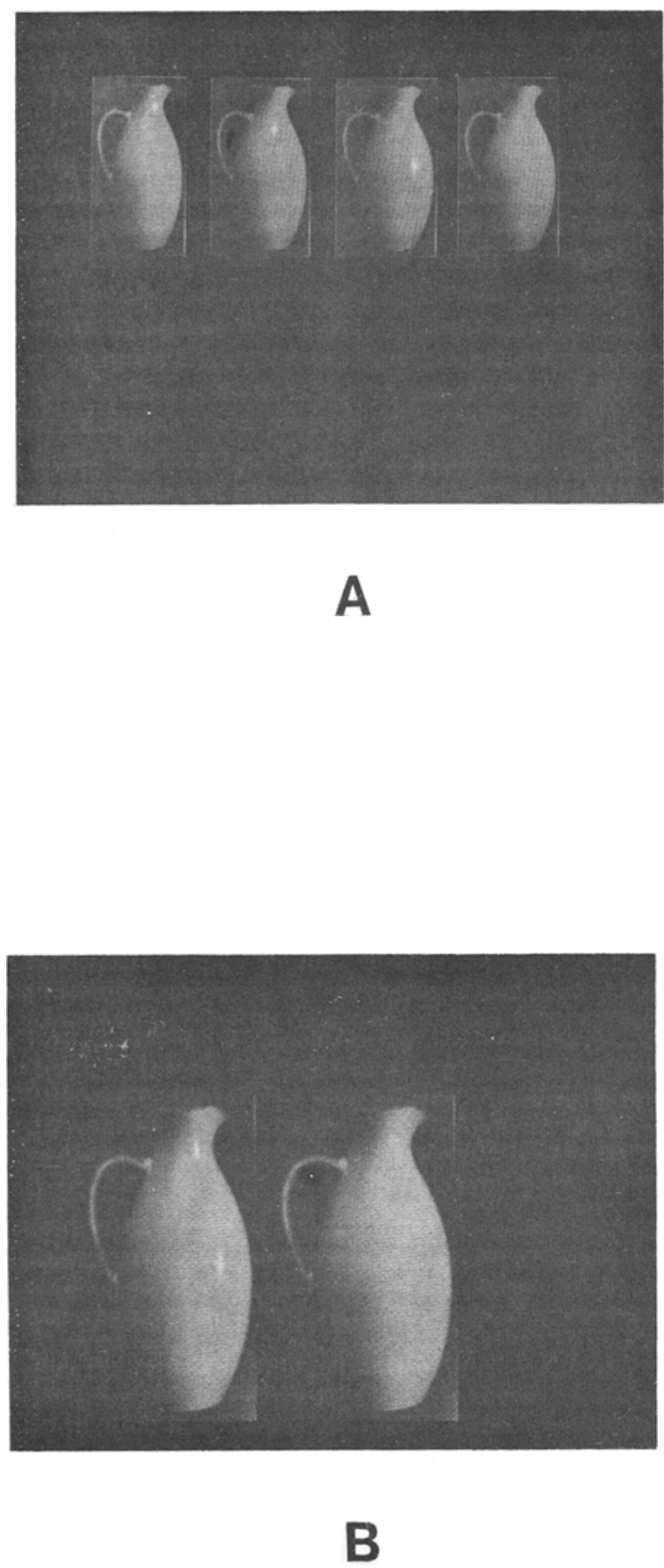

Figure 1. Glossiness produced by highlights. 


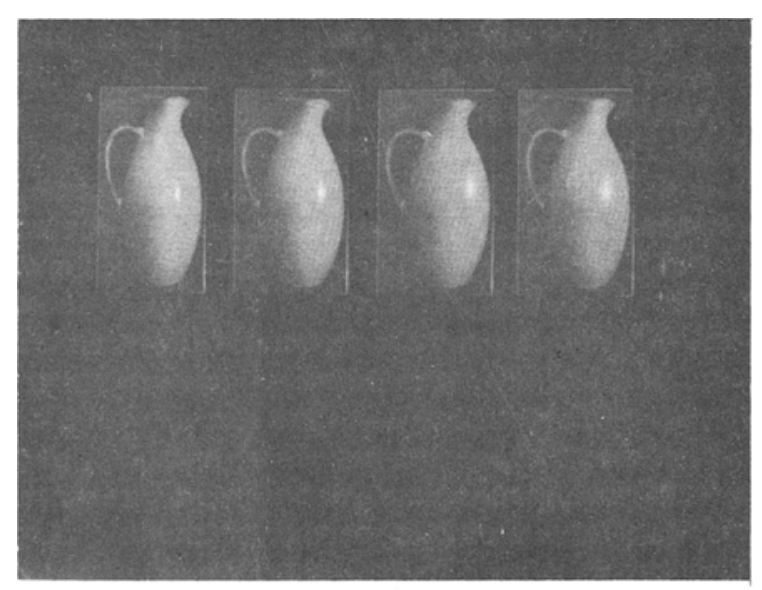

A

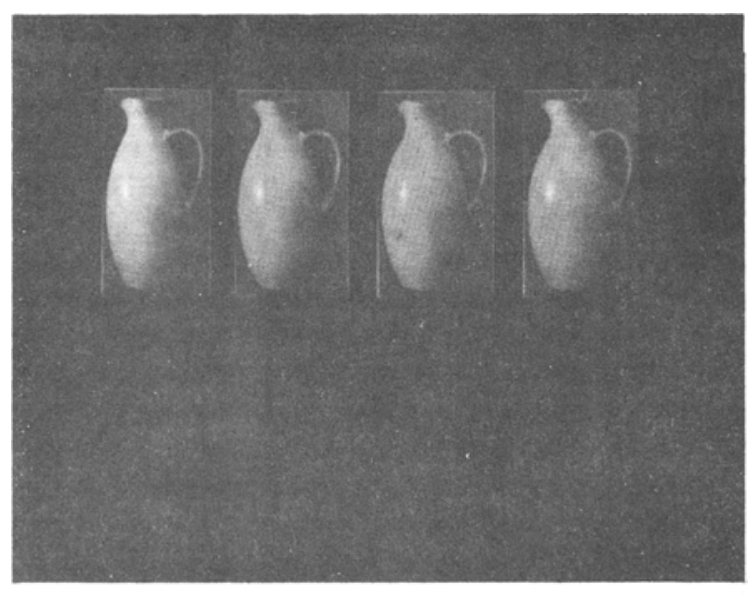

Figure 2. Effects of size and brightness of highlights.

not lead to a perceptual inference about the vase surface that causes the entire vase to appear glossy.

In A of Figure 2 the size of the highlight is increased. In $B$, the brightness of the highlight is increased. Since the size of the highlight in B is constant, the intensity gradient of the highlight is steepened with increasing brightness. Increasing the size and brightness of a highlight increases the area of the vase which looks shiny. The orientation of a highlight is also important. The vertical highlights in A and B of Fig-
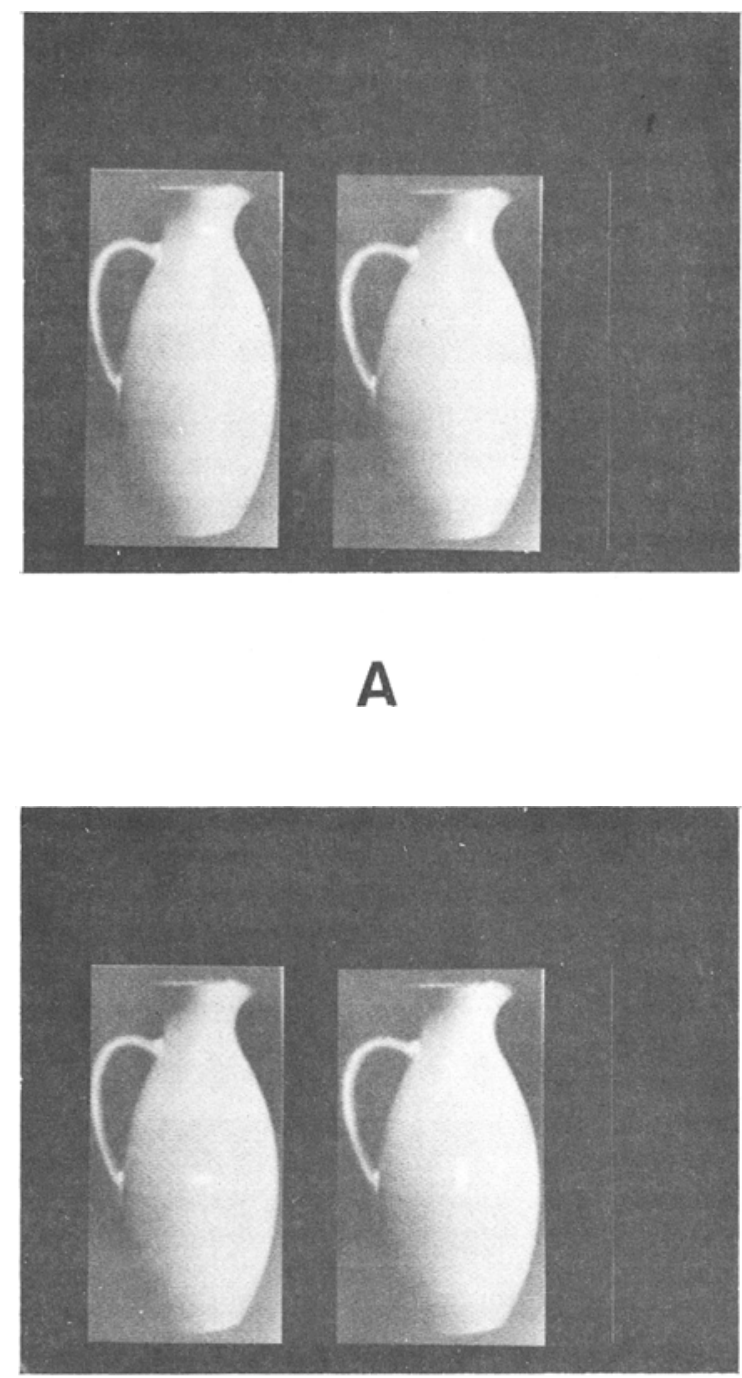

Figure 3. Effect of orientation of highlights.

ure 3 lie in the direction of minimal curvature and are consistent with the information in the picture for the direction of illumination and curvature of the vase surface. The horizontal highlights in $\mathrm{A}$ and $\mathrm{B}$ lie in the direction of maximal curvature and are not consistent with this information. The perception of glossiness produced by the vertical highlights is greater than the perception of glossiness produced by the horizontal highlights. Fifteen observers judged the vase with the vertical highlight in Figure $A$ to be 


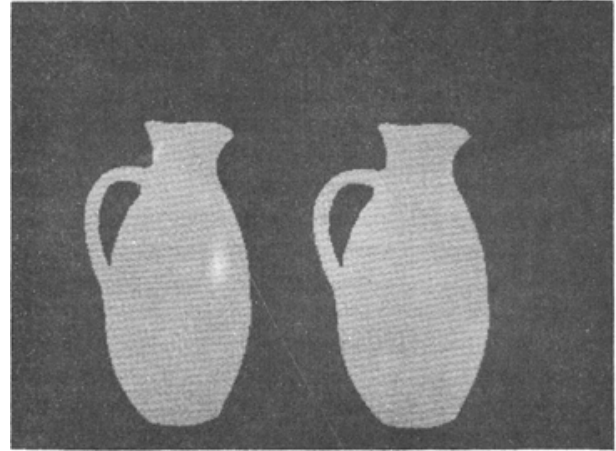

A

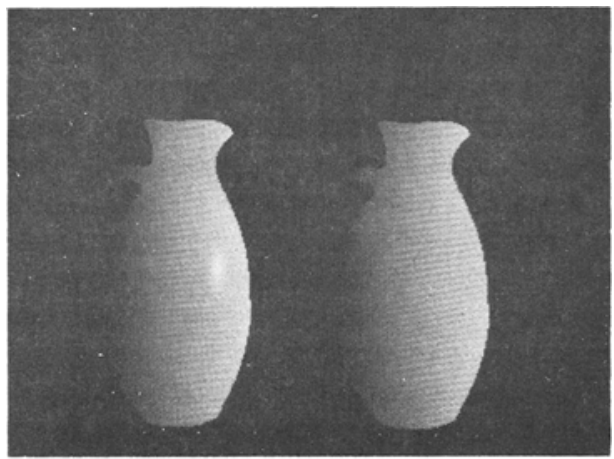

C

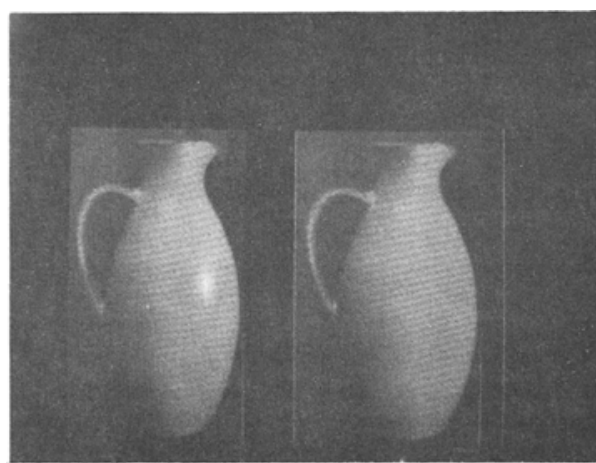

B

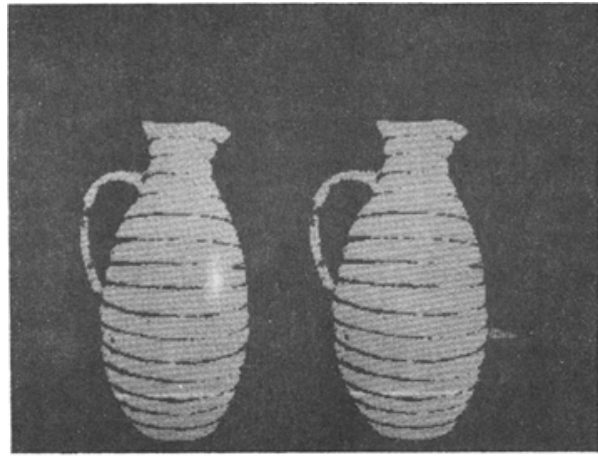

D

Figure 4. Effect of intensity gradient on the vase surface.

more glossy than the vase with the horizontal highlight. Ten observers judged the vase with the vertical highlight in Figure B to be more glossy, while five observers judged the vase with the horizontal highlight to be more glossy.

The outline of the vase was cut from a uniform gray paper and digitized pictures of the gray cutout with and without a highlight are shown in $\mathbf{A}$ of Figure 4. The original digitized pictures of the vase with and without a highlight are shown in B. Comparison of the two pictures shows that a highlight alone fails to produce the perception of glossiness. The perception of glossiness depends not only on the presence of highlights but on the intensity gradient on the surface. In $\mathrm{C}$, a linear intensity gradient decreasing from right to left was superimposed on the gray cutout. The intensity gradient makes the vase surface look curved. Comparison of the highlighted photograph with the unhighlighted photograph shows that the highlight produces the perception of a shinier surface. Several other intensity gradients with the same highlight were tried and produced the perception of glossiness. These observations indicate that the precise intensity gradient is not very important. What appears to be important is for the intensity gradient to produce the perception of a curved surface. The perception of a curved surface can also be evoked by contours, as in D. A highlight superimposed on a curved surface produced by contours (Figure 3D) fails, however, to evoke as clear a perception of glossiness as a highlight on a curved surface produced by an intensity gradient (Figure $3 \mathrm{C}$ ).

These demonstrations suggest that the perception of glossiness is a direct perceptual response to local visual stimulation. It is not the result of a perceptual inference which identifies a surface as reflecting light specularly. As illustrated in Figure 1, highlights that identify the surface as reflecting light specularly do not cause the entire surface to look shiny. Highlights tend to produce a perception of glossiness, as illustrated in Figure 4, if an intensity gradient causes the surface to appear curved. The highlights and inten- 
sity gradient over the surface need not, however, be fully consistent with each other in order to produce the perception of glossiness. The perception of glossiness, however, may be increased, as illustrated in A and $B$ of Figure 3, if they are consistent with each other.

\section{REFERENCE NOTE}

1. Forbus, K. Light source effects. Massachusetts Institute of Technology Artificial Intelligence Laboratory Memo No. 422, 1977.

\section{REFERENCES}

BECK, J. Surface color perception. Ithaca, N.Y: Cornell University Press, 1972.

Graham, C. H., \& Brown, J. L. Color contrast and color appearances: Brightness constancy and color constancy. In $\mathbf{C}$. $\mathbf{H}$. Graham (Ed.), Vision and visual perception. New York: Wiley, 1965.

(Manuscript received June 1, 1980; revision accepted for publication July 30,1981 .) 